Methoden sowie mittels Röntgen-Absorptionsmessungen bestimmt worden war ${ }^{1}$. Dieser Eichbogen wurde zusammen mit zwei Bögen unbekannter Temperaturverteilung (100 A- und $500 \mathrm{~A}$-Bogen) auf der gleichen Platte aufgenommen, deren Schwärzungskurve durch Aufnahme eines Stufensektors bestimmt wurde. Es wurde nun bei einem bestimmten Kathodenabstand das Bild des Eichbogens senkrecht zur Bogenachse photometriert, die Schwärzungswerte in Intensitätswerte umgerechnet und diese auf verschiedene Schichtdicken sich beziehenden Intensitätswerte mittels der ABELschen Integralgleichung in bekannter Weise ${ }^{\mathbf{1}}$ auf die radiale Intensitätsverteilung umgerechnet. Dann wurde die so ermittelte radiale Intensitätsverteilung gegen die aus den früheren Messungen bekannte radiale Tem-

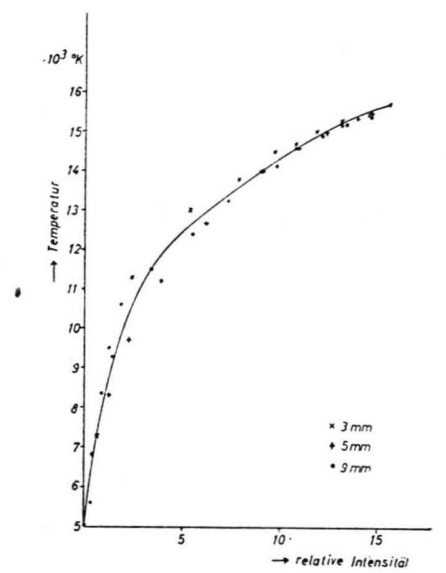

Abb. 2. Abhängigkeit der Gesamtstrahlungsintensität von der

Temperatur. Die Eichpunkte wurden drei verschiedenen

Bogenebenen entnommen.

peraturverteilung aufgetragen. Die so erhaltene Eichkurve (Abb. 2) wurde durch entsprechende Messungen bei anderen Bogenquerschnitten kontrolliert und bestätigt. Die in Abb. 2 eingezeichneten Meßpunkte zeigen die ausgezeichnete innere Übereinstimmung.

Zur Bestimmung der unbekannten Temperaturverteilung der Bögen von 100 und $500 \mathrm{~A}$ wurde in gleicher

^ G. Busz u. W. Finkelnburg, Z. Phys. 139, 212 [1954].
Weise deren radiale Intensitätsverteilung in verschiedenen Abständen von der Kathode ermittelt und aus der Eichkurve (Abb. 2) die entsprechenden Temperaturwerte entnommen. Als Ergebnis zeigt Abb. 3 die Isothermen des Eichbogens (Mitte) und der beiden vorher unbekannten Bögen. Da für unseren Eichbogen oberhalb $16000^{\circ} \mathrm{K}$ keine Eichpunkte mehr zur Verfügung
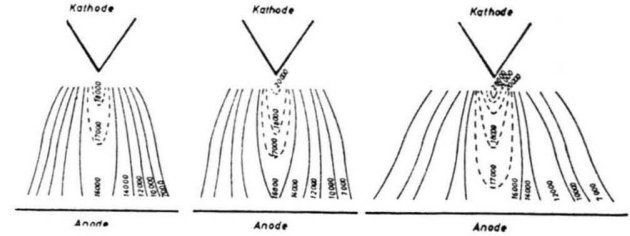

Abb. 3. Isothermen im 100, 200 und 500 A-Argon-Bogen. Die gestrichelten Isothermen wurden aus extrapolierten bzw. interpolierten Kurvenstücken des radialen Temperaturverlaufs erhalten.

standen, wurde die radiale Temperaturverteilung in den drei Beispielen von $16000^{\circ} \mathrm{K}$ bis zur Bogenachse hin extrapoliert. Die aus diesen extrapolierten Kurvenstükken gewonnenen Isothermen sind in $\mathrm{Abb} .3$ gestrichelt eingezeichnet. Für den 500 A-Bogen war zusätzlich aus dem Strahlungsmaximum der Ionenlinien die Isotherme von $28000^{\circ} \mathrm{K}$ bestimmt worden. Eine Erweiterung der behandelten Methode auf den Temperaturbereich oberhalb des ersten Strahlungsmaximums ist dann möglich, wenn empirische Eichwerte zur Verfügung stehen. Dann ist unsere Methode auch brauchbar für den denkbaren Fall, daß einer bestimmten Intensität mehrere Temperaturwerte zuzuordnen sind, da die Temperatur im Bogen in Richtung zur Achse nur zu- und nicht abnehmen kann. - Solche Eichpunkte sind z. B. die Strahlungsmaxima der höheren Ionisationsstufen. Die übliche Bestimmung auf Grund von Linienbreiten und Kontinuum-Intensität versagt in diesem hohen Temperaturbereich, weil die Elektronendichte dann nicht mehr von der Temperatur abhängt.

Herrn Professor W. Finkelnburg danken wir für aufschlußreiche Diskussionen und Fräulein B. Мieth für Hilfe bei den Aufnahmen und Auswertungen.

\section{Zur Erosion aus Metalloberflächen durch Ultraschall-Kavitation}

\author{
Von R. Esche und H. Kimmel
}

Technische Stammabteilung und Forschungslaboratorium der Siemens-Schuckertwerke AG., Erlangen

(Z. Naturforschg. 11 a, 514-515 [1956]; eingegangen am 24. Januar 1956)

Herrn Professor Trendelenburg zum 60. Geburtstag gewidmet

Unter Kavitation versteht man die Bildung von Hohlräumen in Flüssigkeiten. Das Zusammenstürzen dieser Hohlräume erzeugt heftige Druckstöße in der Flüssigkeit. Die durch Kavitation verursachte Zerstörung und
Abtragung von Metalloberflächen ist bereits in zahlreichen Untersuchungen behandelt worden.

Allen diesen Arbeiten ${ }^{1}$ ist die Auffassung gemeinsam, daß die bei Kavitation auftretenden örtlichen Druckund Temperaturspitzen der Größenordnung $10^{3}$ Atm. bzw. $10^{3}{ }^{\circ} \mathrm{K}$ für den Erosionsvorgang verantwortlich sind.

1 Lord Rayleigh, Phil. Mag. (6) 34, 94 [1917]. - B. E. Noltingk u. E. A. Neppiras, Proc. Phys. Soc., Lond. 63, 9, 674 [1950]. - M. Konnfeld u. L. Suvorov, J. Appl. Phys. 15, 495 [1944]. - H. G. Möller u. A. Sсносн, Akust. Z. 6, 165 [1941]. - Tн. Rutenbeck, Z. Metallkde. 33, 4, 145 [1941]. - R. T. KNapp, Proc. Inst. Mech. Engrs. (A) 166, 150 [1952]. 


$$
\text { . }
$$


R. Esche und H. KiмmeL, Zur Erosion aus Metalloberflächen durch Ultraschall-Kavitation (S. 514).

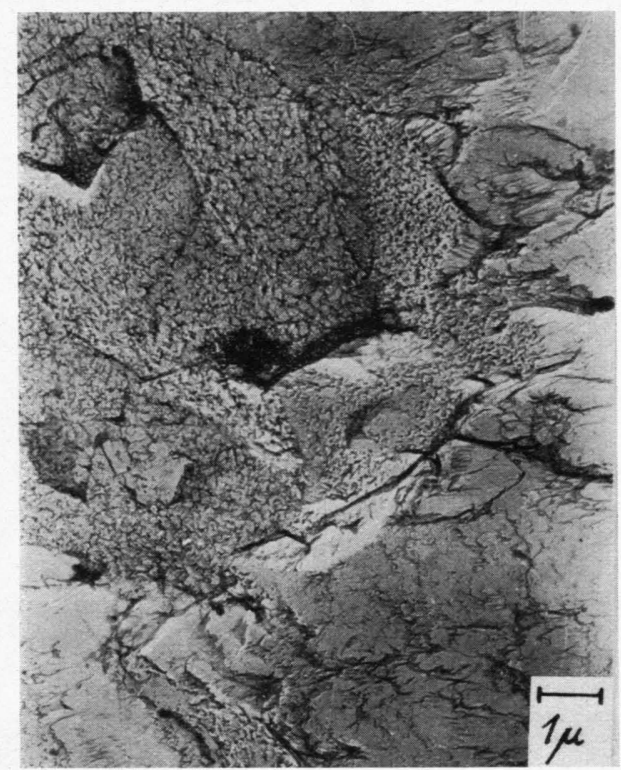

Abb. 1.
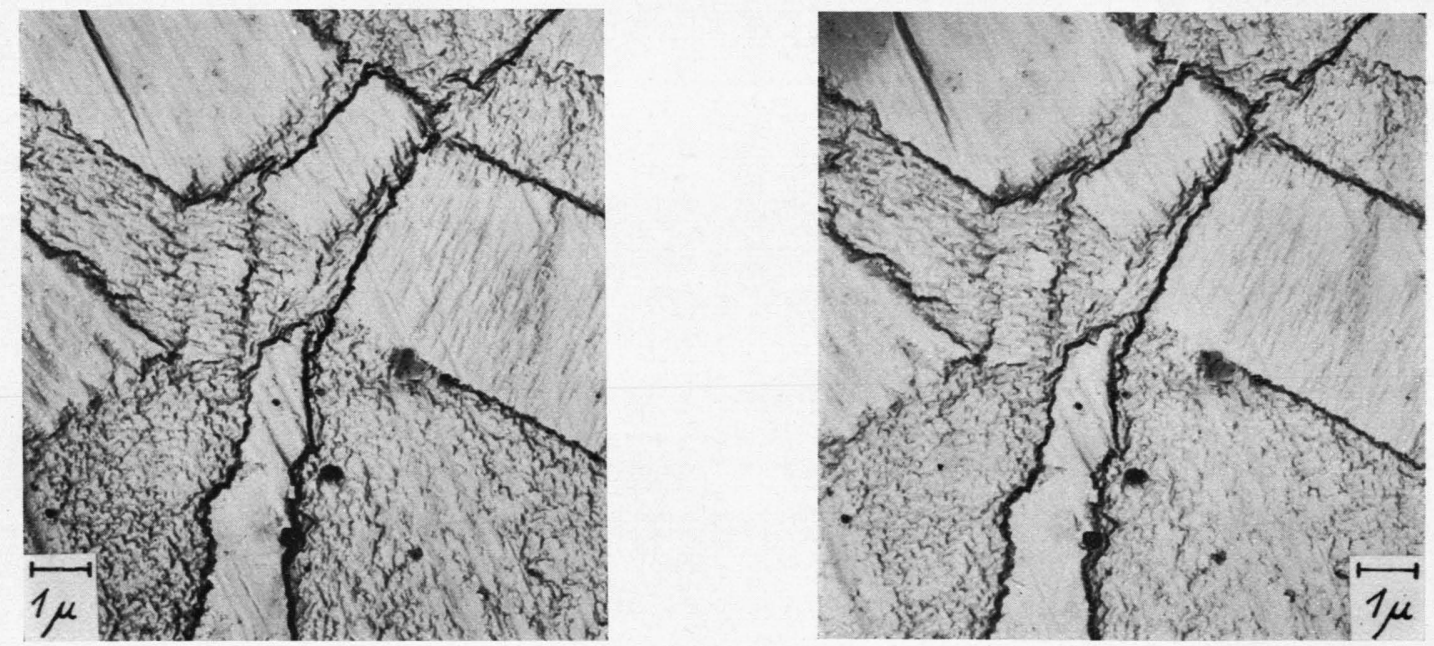

Abb. 2. 
Die Kavitationserosion kann in einfacher Weise mit Ultraschall hervorgerufen werden, da hierbei der zur Kavitationsauslösung erforderliche Energieaufwand besonders gering ist.

In allen Versuchen wurde Kavitation in Wasser ausgelöst, auf besondere Entgasung des Wassers wurde verzichtet in der Annahme, daß zur Erosion die Gasblasenschwingungen mit ihren relativ schwachen Druckspitzen nur unerheblich beitragen. Die Wassertemperatur betrug $20-25^{\circ} \mathrm{C}$. Als Kavitationsgrenzfläche wurde zunächst Aluminium verwendet; es zeigte sich jedoch, daß die harte Oxydhaut das Studium des primären Erosionsvorgangs erschwert. Daher wurden die Untersuchungen an Kupfer weitergeführt.

Da bekanntlich der Kavitationseinsatz von der verwendeten Ultraschall-Frequenz abhängig ist, wurden zunächst verschiedene Frequenzen zur Kavitationsauslösung benutzt $(20,30,430,1200 \mathrm{kHz})$. Bei hohen Frequenzen $(430 \mathrm{kHz})$ wurde ein Quarzschwinger (Schallleistung $300 \mathrm{~W})$ und ein fokussierender Reflektor verwendet. Bei 20 bzw. $30 \mathrm{kHz}$ kamen magnetostriktive bzw. keramische Schwinger zur Anwendung. Die Anordnung wurde so gewählt, daß der Kavitationsangriff möglichst verteilt (,in Schleierform“) auftrat, die Beschallungsstärke und -zeit wurde so gering wie möglich gehalten.

Obwohl die zur Kavitation erforderliche Schallstärke je nach Frequenz sehr verschieden war (z. B. 2-8 W pro $\mathrm{cm}^{2}$ bei $20 \mathrm{kHz}$ und bis zu $500 \mathrm{~W} / \mathrm{cm}^{2}$ bei $430 \mathrm{kHz}$ ), ergab die Untersuchung bei allen Frequenzen das gleiche Erosionsbild, so daß daraufhin stets bei der tiefsten, den kleinsten Energieaufwand erfordernden Ultraschallfrequenz gearbeitet wurde.

Die Beschallungszeit wurde je nach Schallstärke, Frequenz und Art der zu beschallenden Metalloberfläche von $3 \mathrm{sec}$ bis $5 \mathrm{~min}$ gewählt. In einzelnen Versuchen wurde zwischen Ultraschallschwinger und Probe eine schallweiche, dünne Gummimembrane eingebracht, um gegebenenfalls vom Schwinger selbst erodierte Partikelchen vom Erosionsvorgang am Versuchsobjekt auszuschließen. In diesen Fällen mußte länger (1/2 Std.) be-

* Abb. 1 und 2 auf Tafel S. 514 b. schallt werden, um sichtbare Kavitationsspuren am $\mathrm{Ob}$ jekt zu erhalten.

Die Untersuchung der kavitierten Metalloberflächen erfolgte licht- und elektronenmikroskopisch. Bereits bei den ersten Beobachtungen an beschalltem Aluminium fielen deutlich verschiedene mikroskopische Bereiche mit unterschiedlicher Erosion auf (Abb. 1*). An Hand stereoskopischer Aufnahmen ergab sich weiterhin, daß die Strukturen - im wesentlichen handelte es sich dabei um schuppenförmige Gebilde - in Bereichen einheitlichen Kavitationsangriffes eine einheitliche Orientierung aufwiesen. Es wurde daraufhin vermutet, daß die Orientierung der durch den Erosionsvorgang hervorgerufenen Oberflächenstrukturen mit derjenigen des Kristalles in Zusammenhang stehen könnte. Die Folge davon wäre, daß die einheitlich erodierten und orientierten Bereiche mit einzelnen Körnern und die Bereichsgrenzen mit Korngrenzen identisch wären. Die weiteren Untersuchungen an Kupfer bestätigten diese Vermutung vollauf. So wurden z. B. polierte Cu-Flächen leicht angeätzt, um die Korngrenzen sichtbar zu machen und anschließend unter Wasser beschallt. Ein Vergleich der Oberflächenbilder vor und nach der Beschallung ergab, daß sowohl die Materialabtragung durch Kavitation als auch die durch die Erosion verursachten Strukturen von Korn zu Korn verschieden sind (vergl. stereoskopische Abb. 2). Das tiefe Aushöhlen der Korngrenzen wird dadurch verursacht, daß die Kavitation, wie auch aus anderen Untersuchungen hervorgeht, bevorzugt an Rillen angreift. Durch die vorhergegangene Ätzung waren also die Korngrenzen besonders kavitationsempfindlich. Die ungeätzten Proben zeigten nach der Beschallung ein qualitativ gleiches Bild, jedoch waren die Korngrenzen nur noch als Begrenzungslinien von Feldern einheitlicher Strukturorientierung und Erosion sichtbar, nicht mehr als tief eingeschnittene Linien.

Nach den Untersuchungsergebnissen ist somit die kristallographische Orientierung der Körner von Einfluß auf die Stärke der Kavitationserosion. Dies kann auf Unterschiede in der Abtrennarbeit und auch auf verschieden starke Anreicherung von Kavitationskeimen an der Grenzfläche Metall-Flüssigkeit zurückzuführen sein.

\section{Über die Planung und den Bau von Orgeln auf akustischer Grundlage}

Von W. Lottermoser

Physikalisch-Technische Bundesanstalt, Braunschweig

(Z. Naturforschg. 11 a, 515-517 [1956]; eingegangen am 30. April 1956)

Herrn Professor Trendelenburg zum 60. Geburtstag gewidmet

Durch die Anwendung der Oktavsiebanalyse bei Messung der Einschwingvorgänge ${ }^{1}$ und Partialtonzusammensetzungen ${ }^{2}$ von Orgelklängen war es möglich, die

1 F. Trendelenburg, E. Thienhaus u. E. Franz, Akust. Z. 1, 59 [1936]; 3, 7 [1938].
Ursachen für die hervorragende Klangwirkung der wertvollen Barockorgeln aufzuklären. Nachdem eine Reihe neuer und alter Orgeln verschiedener Typen ${ }^{3}$ untersucht worden war, interessierte die Aufgabe, modernen Orgeln durch akustische Planung und Abgleichung ähnliche Klangqualitäten $\mathrm{zu}$ verleihen, wie sie die hochwertigen Instrumente besitzen.

Um dieses Ziel zu erreichen, können zwei Wege beschritten werden: Entweder ist die Raumakustik als gegeben hinzunehmen und die Orgel an den Raum anzupassen oder aber sind die Mensuren der Orgel als gegeben zu betrachten und die Raumakustik auf die

2 W. Lottermoser, Z. Naturforschg. 5 a, 159 [1950].

3 W. Lottermoser, Acustica Beih. 3, 129 [1953]. 